\title{
Ultrafast Magnetism of a Ferrimagnet across the Spin-Flop Transition in High Magnetic Fields
}

\author{
J. Becker ${ }^{1,2}$ A. Tsukamoto, ${ }^{3}$ A. Kirilyuk, ${ }^{1}$ J. C. Maan, ${ }^{4}$ Th. Rasing, ${ }^{1}$ P. C. M. Christianen, ${ }^{4}$ and A. V. Kimel ${ }^{1}$ \\ ${ }^{1}$ Radboud University, Institute for Molecules and Materials, Nijmegen 6525 AJ, The Netherlands \\ ${ }^{2}$ High Field Magnet Laboratory (HFML-EMFL), Radboud University, Toernooiveld 7, 6525 ED Nijmegen, The Netherlands \\ ${ }^{3}$ College of Science and Technology, Nihon University, 24-1 Narashinodai 7-chome, Funabashi-shi, Chiba 274-8501, Japan \\ ${ }^{4}$ High Field Magnet Laboratory (HFML-EMFL), Radboud University, Toernooiveld 7, 6525 ED NIJMEGEN, The Netherlands
}

(Received 19 October 2016; revised manuscript received 13 January 2017; published 14 March 2017)

\begin{abstract}
We show that applying magnetic fields up to $30 \mathrm{~T}$ has a dramatic effect on the ultrafast spin dynamics in ferrimagnetic GdFeCo. Upon increasing the field beyond a critical value, the dynamics induced by a femtosecond laser excitation strongly increases in amplitude and slows down significantly. Such a change in spin response is explained by different dynamics of the Gd and FeCo magnetic sublattices following a spin-flop phase transition from a collinear to a noncollinear spin state.
\end{abstract}

DOI: 10.1103/PhysRevLett.118.117203

Ultrafast magnetism gained widespread attention by the seminal observation of ultrafast laser-induced demagnetization of metallic Ni [1]. This experiment revealed that exciting a ferromagnet by femtosecond laser pulses causes a partial collapse of magnetic order, occurring much faster than could be explained by the interactions responsible for angular momentum exchange with the spin system that were known at the time. The field was further fueled by experiments revealing that femtosecond laser excitations open up effective channels of angular momentum transfer and can lead to all-optical magnetic switching [2-5]. Controlling the amplitude and time scale of the response of spins to a femtosecond laser excitation has become a topic crossing the borders of the subfield of ultrafast magnetism, influencing research on magnetic recording [2], spintronics [6], and magnonics [7].

It has been demonstrated that the time scale of the spin response to a femtosecond laser excitation can be controlled by varying the Curie temperature, the atomic magnetic moment of the chemical elements, and the intensity of the excitation [8-10]. The question, whether high magnetic fields can also affect the time scale of the ultrafast spin response to a femtosecond laser excitation, has not been answered yet. A simple estimate shows that, as the period of the Larmor precession of the electron spin is $28 \mathrm{GHz} / \mathrm{T}$, in order to affect the spin response in the range 1-10 ps, one has to apply magnetic fields above $10 \mathrm{~T}$. However, combining high magnetic fields with ultrafast magnetization dynamics experiments has so far been a challenge.

We have built a setup that allows us to investigate laserinduced ultrafast magnetization dynamics in high magnetic fields up to 37.5 T. Here, we report time resolved magnetooptical Kerr effect (TR MOKE) measurements in magnetic fields up to $30 \mathrm{~T}$ of the rare earth transition metal (RE TM) alloy $\mathrm{GdFeCo}$. We find that high fields surprisingly lead to an increased susceptibility of the system to an external laser stimulus and cause an initial slowing down, rather than speeding up, of the ultrafast magnetization dynamics. To explain the observed dynamics in the uncompensated ferrimagnet, we suggest a phenomenological model which is based on substantially different dynamics of the RE and TM magnetic sublattices.

In the studied amorphous ferrimagnetic alloy $\mathrm{GdFeCo}$, the antiferromagnetic exchange coupling between the RE and the TM magnetic sublattices favors a collinear antiparallel alignment. The magnetizations of the transition metal $M_{T}$ and the rare earth $M_{R}$ sublattices show different temperature behavior. The studied alloy has a composition of $24 \% \mathrm{Gd}, 66.5 \% \mathrm{Fe}$, and $9.5 \% \mathrm{Co}$ which results in a compensation temperature $T_{M}=283 \mathrm{~K}$ at which the sublattices cancel each other out, leading to a zero net magnetization $M_{\text {net }}=\left|M_{R}-M_{T}\right|=0$. Below this temperature $T<T_{M}, M_{\text {net }}$ is dominated by the RE sublattice $M_{R}>M_{T}$. Above the compensation $T>T_{M}$, the situation is opposite $M_{R}<M_{T}$. The Curie temperature of the alloy is around $500 \mathrm{~K}$. The studied sample is a multilayer structure grown on a $\mathrm{SiO}_{2}$ substrate: $\mathrm{SiO}_{2} / \mathrm{AlTi}(10) / \mathrm{SiN}(5) /$ $\operatorname{RETM}(20) / \operatorname{SiN}(60)$, where the layer thickness is given in parentheses in $\mathrm{nm}$. The GdFeCo magnetic layer in this structure is characterized by an easy-axis type of magnetic anisotropy, oriented perpendicularly to the sample plane. The anisotropy is relatively weak in $\mathrm{GdFeCo}$ due to the $S$-state $\mathrm{Gd}^{3+}$ ions $[11,12]$.

If the magnetic field reaches the critical value $H_{\mathrm{sf}}$, a ferrimagnet undergoes a spin-flop transition [13,14]; i.e., the magnetizations of the sublattices turn from the normal of the sample, get canted, and form a noncollinear state. The spin-flop transition is shown schematically in Fig. 1(a), where $H_{\text {sf }}$ marks the boundary between the collinear region I and the noncollinear region II. The canting angles $\theta_{R, T}$ of the magnetic sublattices can be further controlled by the 
(a)

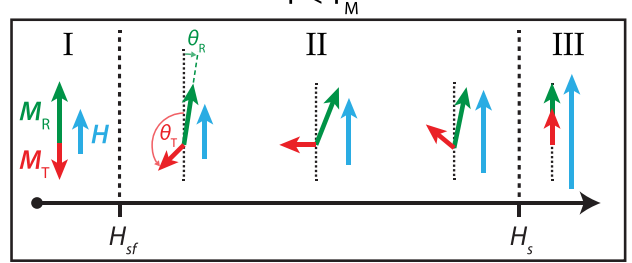

(b)

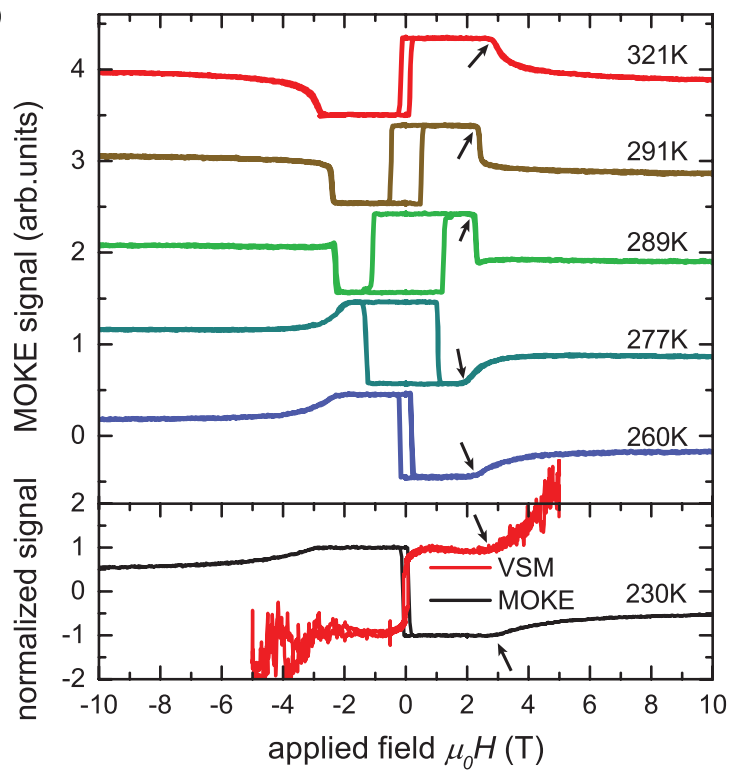

(c)

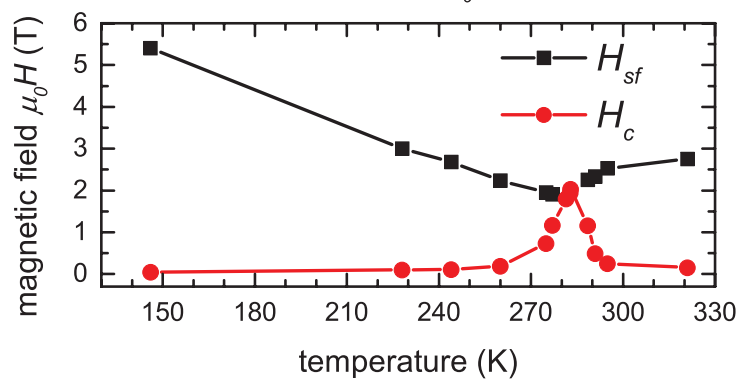

FIG. 1. (a) Schematic representation of the spin-flop behavior at $T<T_{M}$ of the rare earth and transition metal magnetic sublattices $\left(M_{R}\right.$ and $M_{T}$, respectively) upon increasing the external magnetic field (adapted from [15]). (b) Static MOKE data of the GdFeCo sample measured at $630 \mathrm{~nm}$ wavelength at different temperatures from 230.0 to $321.0 \mathrm{~K}$. A paramagnetic background was subtracted from the measurements. The magnetization compensation temperature $T_{M}$ lies at around $283 \mathrm{~K}$. MOKE and VSM measurement are shown for $230 \mathrm{~K}$. (c) Coercive field $H_{c}$ and spin-flop field $H_{\text {sf }}$ extracted from the static MOKE data as a function of temperature. The error lies within the symbol size.

magnetic field $H$. Increasing $H$ beyond $H_{\text {sf }}$ in region II, both sublattice magnetizations will tilt towards $\boldsymbol{H}$ as it competes more and more with the intersublattice exchange interaction. If the magnetic anisotropy is weak, the spin-flop field can be approximated by $[13,16] H_{\mathrm{sf}} \sim$ $\lambda_{R-T}\left|M_{R}-M_{T}\right|$, with $\lambda_{R-T}$ the intersublattice exchange field parameter.
At the saturation field $H_{S} \sim \lambda_{R-T}\left(M_{R}+M_{T}\right)$, which defines the onset of region III, both magnetizations align collinearly along $\boldsymbol{H} . H_{S}$ is of the order of $\sim 100 \mathrm{~T}$ for GdFeCo [17] as it has to overcome the intersublattice exchange field.

To define the spin-flop fields for the studied structure we measured the polar magneto-optical Kerr effect (MOKE) at normal incidence of light at a wavelength of $630 \mathrm{~nm}$. In this experiment, the light probes the normal component of the magnetization of the FeCo sublattice $[18,19]$. Figure 1(b) shows the results of these measurements. Besides hysteresis loops that close at the coercive fields $H_{C}$, the measurements reveal the spin-flop transition at fields $>2 \mathrm{~T}$, indicated by the black arrows in Fig. 1(b). The spin-flop fields $H_{\mathrm{sf}}$ and the coercive fields $H_{C}$ deduced from these data are plotted in Fig. 1(c). $H_{\text {sf }}$ indeed decreases along with $\left|M_{R}-M_{T}\right|$ upon approaching $T_{M}$. As a consequence of the magnetic reorientation during the spin-flop transition in ferrimagnets, a drastic increase of the net magnetization upon reaching $H_{\mathrm{sf}}[13,16]$ can be observed using a vibrating sample magnetometer (VSM) [see red curve at $230 \mathrm{~K}$ in Fig. 1(b)].

Figure 2 shows a set of TR MOKE measurements on GdFeCo below $\left(T=230 \mathrm{~K}, \mu_{0} H_{\mathrm{sf}}=3.0 \mathrm{~T}\right)$ and above $\left(T=289 \mathrm{~K}, \mu_{0} H_{\mathrm{sf}}=2.4 \mathrm{~T}\right) T_{M}$ at different applied magnetic fields. All measurements were performed with $630 \mathrm{~nm}$ probe and $800 \mathrm{~nm}$ pump wavelengths in the polar Kerr geometry. As in the static MOKE measurements, the probe at this wavelength is mainly sensitive to the polar component of the TM sublattice magnetization. It is seen that crossing $H_{\text {sf }}$ has a significant influence on the laserinduced magnetization dynamics. For both temperatures, the demagnetization at fields below $H_{\text {sf }}$ is about $4 \%$ of the ferrimagnetic saturation magnetization $M_{T}\left(H_{C}<H<\right.$ $\left.H_{\text {sf }}\right)$ and occurs on a subpicosecond time scale. In stark contrast, above $H_{\mathrm{sf}}$ in region II the amplitude and time scale of the transient signal increase by an order of magnitude. As the field is increased further beyond $H_{\mathrm{sf}}$, the amplitude is reduced again and the signal peaks at earlier delay times. Additionally, oscillations appear at fields above $H_{\mathrm{sf}}$ that increase in frequency as the field is increased.

Furthermore, for $T>T_{M}$, a change in sign of the transient signal for fields above compared to fields below $H_{\text {sf }}$ takes place. This can be connected to a similar change in sign visible in the static MOKE measurements at the same temperature and field shown in Fig. 1(b). This asymmetry in signal with respect to temperature relative to $T_{M}$ has been observed before in ferrimagnets [20] and was linked to slight variations in the chemical composition of the GdFeCo layer. However, comparing all measurements presented in Fig. 2 to the static MOKE data at the same temperatures, we can conclude that the femtosecond laser excitation always causes an initial reduction of the polar component of the TM magnetization. 

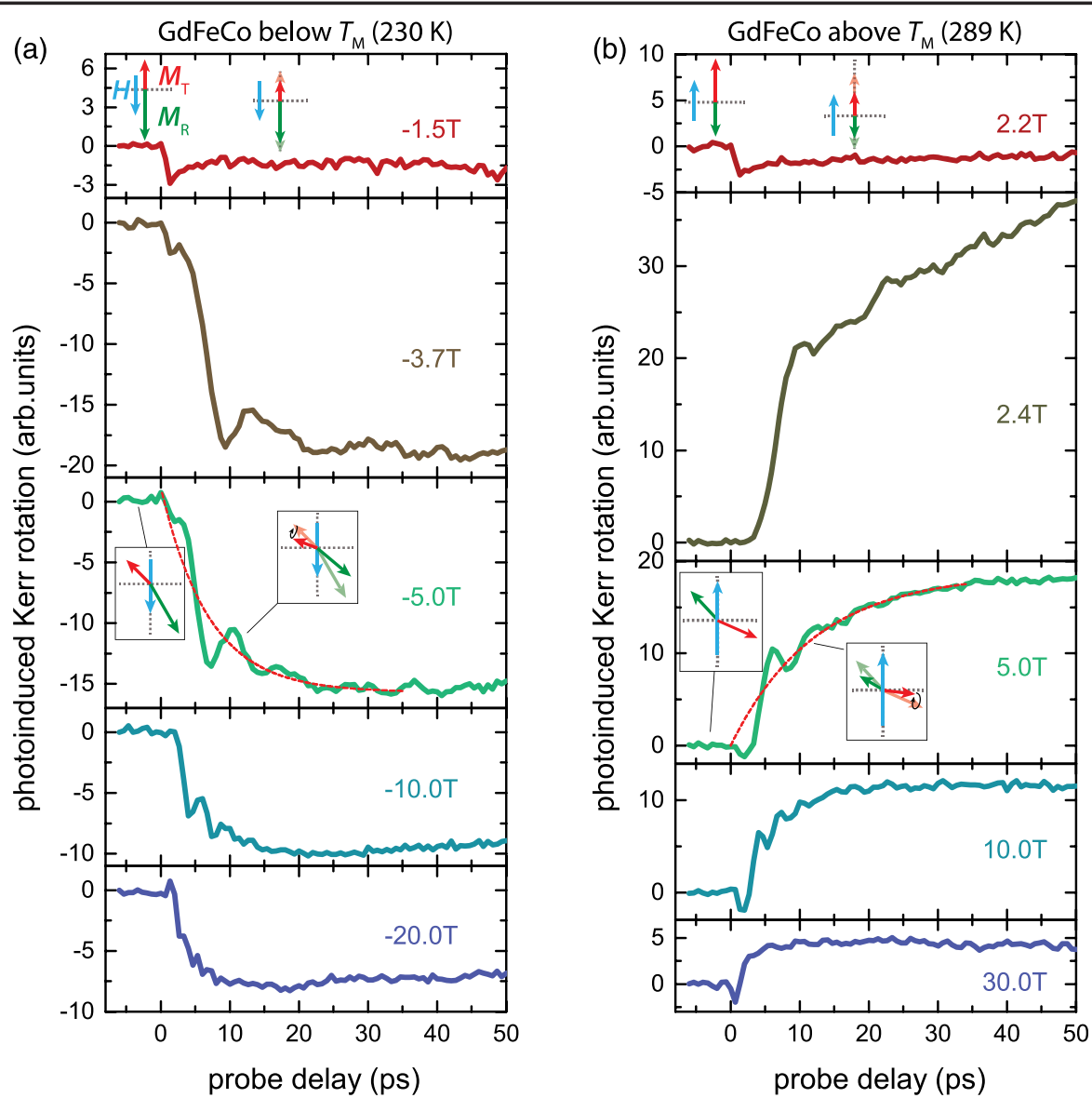

FIG. 2. (a) and (b) show the magnetization dynamics measured by TR MOKE in GdFeCo at different applied magnetic fields below and above $T_{M}$, respectively. As an example, we show the exponential fit (red dashed line) used to quantify the dynamics in the first picoseconds after pump arrival for both temperatures at $5 \mathrm{~T}$. Also at $5 \mathrm{~T}$ and in the first panel for fields $<H_{\text {sf }}$, the position of the rare earth (green) and transition metal (red) magnetic sublattice is depicted schematically for two cases, probe delay $<0$ and $>0$. In the measurements at $1.5 \mathrm{~T}$ and $230 \mathrm{~K}$ the signal slightly decreases in the range between $40-50 \mathrm{ps}$. Such a slight decrease is most likely an artifact due to instabilities of the sample temperature and laser intensity. Note that ferrimagnets are especially sensitive to such instabilities in the vicinity of the compensation temperature and spin-flop transitions.

Fitting the data of the first picoseconds after the arrival of the pump stimulus with a single exponential function of the form $A \exp [(-x / \tau)]$, allows us to find the amplitude $A$ and decay time $\tau$ [dashed red lines in Figs. 2(a) and 2(b) at 5 T]. In Figs. 3(a) and 3(c), the parameters $|A|$ and $\tau$ are shown as a function of the applied field. At both temperatures, $|A|$ and $\tau$ show similar behavior: a sharp increase at $H_{\mathrm{sf}}$, followed by a continuous decrease as the field is further increased.

The frequencies of the oscillations were determined by subtracting the exponential fit from the data and fitting the remainder with a damped sine function (a comparative FFT analysis can be found in the Supplemental Material [21]). The deduced frequencies are plotted in Figs. 3(b) and 3(d). The characteristic time scale of the oscillations is $1 / \omega=1 /(2 \pi f) \sim 1 \mathrm{ps}$, which is an order of magnitude smaller than the decay time of the exponential fit $\tau \sim 10 \mathrm{ps}$.

The equilibrium orientation of the TM spins, probed in our experiment, is defined by the minimum of the thermodynamic potential which, disregarding the demagnetizing fields, is given by

$U=-\mu_{0} \boldsymbol{H}\left(\boldsymbol{M}_{R}+\boldsymbol{M}_{T}\right)+\lambda_{R-T} \mu_{0} \boldsymbol{M}_{R} \boldsymbol{M}_{T}+U_{a(R)}+U_{a(T)}$,

with the first term representing the Zeeman energy, the second the exchange energy, and the third and fourth terms the anisotropy energy for the rare earth and transition metal sublattice, respectively. The anisotropy energy is given by $U_{a(R, T)}=K_{u}^{R, T} \sin ^{2}\left(\theta_{R, T}\right)$ [16], with $K_{u}^{R, T}$ the uniaxial anisotropy constant for the respective sublattice. It is known that a femtosecond laser pulse can cause demagnetization of the TM sublattice on a characteristic time scale $<1 \mathrm{ps}$, demagnetization of the Gd sublattice on a time scale of $10 \mathrm{ps}$ and a change of the magnetic anisotropy constant on a time scale determined by the electron-phonon interaction $(<3$ ps) [9,22-24]. Such laser-induced changes 

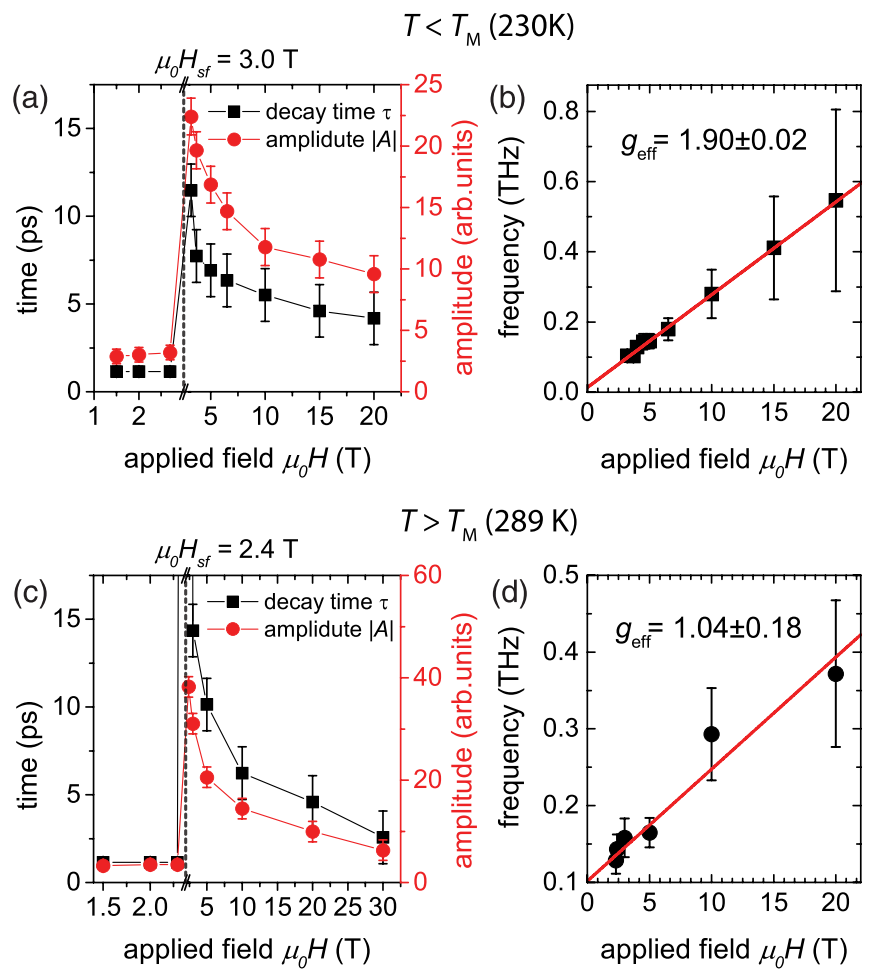

FIG. 3. Absolute value of the amplitude $|A|$ (red circles) and decay time $\tau$ (black squares) of the single-exponential fits to the data in Fig. 2 are shown for the measurement below (a) and above $T_{M}$ (c). (b) and (d) show the extracted frequencies from the same oscillations as a function of applied magnetic field. The lines (red) are linear fits of the data according to Eq. (2).

can alter the equilibrium orientation of the spins and may trigger spin resonances in magnetic media, provided that the magnetization and the applied magnetic field are not collinear $[25,26]$.

Therefore, the oscillations observed in the experiment at fields $\geq H_{\text {sf }}$ can be assigned to the so called spin-flop resonance $\omega_{\mathrm{sf}}$, which in asymptotic approximation is a linear function of the applied field. Its slope can be expressed as follows, assuming that the magnetic anisotropy is small compared to the applied magnetic field [15]:

$$
\frac{d \omega_{\mathrm{sf}}}{d H} \sim \gamma_{\mathrm{eff}} \mu_{0}, \quad \gamma_{\mathrm{eff}}=\frac{M_{R}-M_{T}}{M_{R} / \gamma_{R}-M_{T} / \gamma_{T}},
$$

with $H$ the applied magnetic field and $\gamma_{\text {eff }}$ the effective gyromagnetic ratio and $\gamma_{R, T}$ the gyromagnetic ratios of the RE and TM sublattice, respectively. Figures 3(b) and 3(d) show the frequency versus field relationship for the observed oscillations at 230 and $289 \mathrm{~K}$, respectively. Using Eq. (2) to fit the data, the following effective $g$ factors $g_{\text {eff }}=\hbar \gamma_{\text {eff }} / \mu_{b}$ are obtained: $g_{\text {eff }}=1.04 \pm 0.18$ at $289 \mathrm{~K}$ and $g_{\text {eff }}=1.90 \pm 0.02$ at $230 \mathrm{~K}$.

The difference between the values can be explained by the temperature dependence of the effective gyromagnetic ratio $\gamma_{\text {eff }}$. As $\gamma_{R} \neq \gamma_{T}, \gamma_{\text {eff }}$ depends sensitively on $M_{R}-M_{T}$ which leads to a strong temperature dependence of $g_{\text {eff }}$ around $T_{M}$ that has been theoretically described and observed before [27-31]. At $230 \mathrm{~K}, g_{\text {eff }}$ is close to the Gd $g$ factor of 2.0 [11]. The value of $g_{\text {eff }}$ at $289 \mathrm{~K}$ is much smaller, which can be explained by being much closer to $T_{M}=283 \mathrm{~K}$ at which $g_{\mathrm{eff}}=0$ (see Supplemental Material [21] for an additional measurement at $295 \mathrm{~K}$ ).

The observed precessions can be triggered only if the equilibrium orientation of the magnetization changes much faster than the period of the oscillations, which have a characteristic time scale of $1 / \omega \sim 1$ ps. Consequently, only the ultrafast demagnetization of the TM magnetization can be the trigger of these oscillations.

In our TR MOKE experiment we probe the polar component of the TM magnetization. While in the collinear phase in region I the magneto-optical signal is due to the longitudinal dynamics of the TM magnetization only, the observed jump in amplitude $|A|$ upon crossing $H_{\text {sf }}$ can be caused by the already mentioned change in equilibrium orientation of the magnetizations caused by a laser-induced change in the thermodynamic potential. The dynamics described by the exponential function in Fig. 2 occurs on a time scale $\tau$ of tens of picoseconds, much slower than what is expected in transition metals, either for laserinduced ultrafast demagnetization or changes in anisotropy [23]. The only process which can account for dynamics on such long time scales, is the laser-induced demagnetization of the Gd sublattice $[9,24]$. In the canted state, this will also cause changes in the TM sublattice orientation, due to the RE TM exchange interaction. At increasing fields $H>H_{\text {sf }}$ this dynamics is gradually suppressed. This is consistent with the here-presented model: as $H$ competes more with the intersublattice exchange field, the magnetic structure becomes more collinear, lessening the effect of the $\mathrm{Gd}$ dynamics on the polar TM sublattice dynamics. At the saturation field $H_{S}$ at which all sublattice magnetizations align collinearly with the external field, one should again observe only the ultrafast demagnetization of the TM magnetization such as at fields $H<H_{\text {sf. }}$. Thus, in the noncollinear state, the magnetic field can be used to control the time scale of the measured magnetization dynamics through canting the magnetic sublattices.

To conclude, our setup for high-field ultrafast spectroscopy allows us to reveal the impact of magnetic fields, comparable with that of the exchange interaction, on the magnetization dynamics in the amorphous heavy RE TM alloy GdFeCo. By applying fields of different strengths we can switch between different regimes of magnetization dynamics: Below the spin-flop field in the collinear state, only the ultrafast demagnetization of the TM sublattice is observed. In the noncollinear state, the laser-induced magnetization dynamics is strongly field dependent up to $30 \mathrm{~T}$ and the data suggest that both magnetic sublattices distinctly affect the transient magneto-optical signal: The 
subpicosecond response of the TM magnetic sublattice triggers magnetic resonance oscillations in the $\mathrm{THz}$ range, while the RE sublattice response causes much slower magnetization dynamics on the time scale of tens of picoseconds. Following up on our demonstration, the challenge for further experimental and theoretical work is to understand and describe in detail the effect of noncollinear spins on the ultrafast magnetization dynamics in multisublattice systems. In particular, the role of the RE-magnetic sublattice and the influence of anisotropy in samples with high spin-orbit interaction are of significant interest.

The authors would like to thank P. Albers, A. Toonen, and A. van Etteger for technical support. We especially thank Dr. M. Huijben at the University of Twente for performing the VSM measurements. This research was partially supported by de Nederlandse Organisatie voor Wetenschappelijk Onderzoek (NWO), de Stichting voor Fundamenteel Onderzoek der Materie (FOM), the European Union's Seventh Framework Program (FP7/ 2007-2013) Grant No. 281043 (Femtospin), and the European Union's Seventh Framework Program (FP7/ 2007-2013)/ERC Grant Agreement No. 257280 (Femtomagnetism) and Grant Agreement No. 339813 (Exchange). Part of this work was also supported by EuroMagNET II under the EU Contract No. 228043. We acknowledge the support of the HFML-RU/FOM, member of the European Magnetic Field Laboratory (EMFL).

[1] E. Beaurepaire, J.-C. Merle, A. Daunois, and J.-Y. Bigot, Phys. Rev. Lett. 76, 4250 (1996).

[2] C. D. Stanciu, F. Hansteen, A. V. Kimel, A. Kirilyuk, A. Tsukamoto, A. Itoh, and T. Rasing, Phys. Rev. Lett. 99, 047601 (2007).

[3] T. Ostler et al., Nat. Commun. 3, 666 (2012).

[4] C.-H. Lambert, S. Mangin, B. S. D. Ch. S. Varaprasad, Y. K. Takahashi, M. Hehn, M. Cinchetti, G. Malinowski, K. Hono, Y. Fainman, M. Aeschlimann, and E. E. Fullerton, Science 345, 1337 (2014).

[5] S. Mangin, M. Gottwald, C.-H. Lambert, D. Steil, V. Uhl, L. Pang, M. Hehn, S. Alebrand, M. Cinchetti, G. Malinowski et al., Nat. Mater. 13, 286 (2014).

[6] J. Walowski and M. Münzenberg, J. Appl. Phys. 120, 140901 (2016).

[7] B. Lenk, H. Ulrichs, F. Garbs, and M. Münzenberg, Phys. Rep. 507, 107 (2011).

[8] B. Koopmans, J. J. M. Ruigrok, F. Dalla Longa, and W. J. M. de Jonge, Phys. Rev. Lett. 95, 267207 (2005).

[9] B. Koopmans, G. Malinowski, F. Dalla Longa, D. Steiauf, M. Fähnle, T. Roth, M. Cinchetti, and M. Aeschlimann, Nat. Mater. 9, 259 (2009).
[10] T. A. Ostler, R. F. L. Evans, R. W. Chantrell, U. Atxitia, O. Chubykalo-Fesenko, I. Radu, R. Abrudan, F. Radu, A. Tsukamoto, A. Itoh, A. Kirilyuk, T. Rasing, and A. Kimel, Phys. Rev. B 84, 024407 (2011).

[11] J. Jensen and A. R. Mackintosh, Rare Earth Magnetism (Clarendon, Oxford, 1991).

[12] J. M. Coey, Magnetism and Magnetic Materials (Cambridge University Press, Cambridge, 2010).

[13] A. E. Clark and E. Callen, J. Appl. Phys. 39, 5972 (1968).

[14] A. K. Zvezdin, I. A. Lubashevsky, R. Z. Levitin, G. M. Musaev, V. V. Platonov, and O. M. Tatsenko, Itinerant Electron Magnetism: Fluctuation Effects (Springer, Dordrecht, 1998), Chap. 16, pp. 285-302.

[15] A. G. Gurevich and G. A. Melkov, Magnetization Oscillations and Waves (CRC Press, Boca Raton, 1996).

[16] R. Radwanski, Physica B+C (Amsterdam) 142, 57 (1986).

[17] T. Beuerle, M. Liebs, K. Hummler, and M. Fähnle, J. Magn. Magn. Mater. 132, L1 (1994).

[18] J. K. Lang, Y. Baer, and P. A. Cox, J. Phys. F 11, 121 (1981).

[19] E. Arenholz, E. Navas, K. Starke, L. Baumgarten, and G. Kaindl, Phys. Rev. B 51, 8211 (1995).

[20] M. Amatsu, S. Honda, and T. Kusuda, IEEE Trans. Magn. 13, 1612 (1977).

[21] See Supplemental Materal at http://link.aps.org/ supplemental/10.1103/PhysRevLett.118.117203 for details on the data analysis of the oscillations and additional measurements at $295 \mathrm{~K}$.

[22] U. Bovensiepen, J. Phys. Condens. Matter 19, 083201 (2007).

[23] A. Kirilyuk, A. V. Kimel, and T. Rasing, Rev. Mod. Phys. 82, 2731 (2010).

[24] I. Radu, K. Vahaplar, C. Stamm, T. Kachel, N. Pontius, H. A. Dürr, T. A. Ostler, J. Barker, R. F. L. Evans, R. W. Chantrell, A. Tsukamoto, A. Itoh, A. Kirilyuk, T. Rasing, and A. V. Kimel, Nature (London) 472, 205 (2011).

[25] M. van Kampen, C. Jozsa, J. T. Kohlhepp, P. LeClair, L. Lagae, W. J. M. de Jonge, and B. Koopmans, Phys. Rev. Lett. 88, 227201 (2002).

[26] E. Carpene, E. Mancini, D. Dazzi, C. Dallera, E. Puppin, and S. De Silvestri, Phys. Rev. B 81, 060415 (2010).

[27] R. F. Soohoo and A. H. Morrish, J. Appl. Phys. 50, 1639 (1979).

[28] C. D. Stanciu, A. V. Kimel, F. Hansteen, A. Tsukamoto, A. Itoh, A. Kirilyuk, and T. Rasing, Phys. Rev. B 73, 220402 (R) (2006).

[29] M. Binder, A. Weber, O. Mosendz, G. Woltersdorf, M. Izquierdo, I. Neudecker, J. R. Dahn, T. D. Hatchard, J.-U. Thiele, C. H. Back, and M. R. Scheinfein, Phys. Rev. B 74, 134404 (2006).

[30] T. Kato, K. Nakazawa, R. Komiya, N. Nishizawa, S. Tsunashima, and S. Iwata, IEEE Trans. Magn. 44, 3380 (2008).

[31] F. Schlickeiser, U. Atxitia, S. Wienholdt, D. Hinzke, O. Chubykalo-Fesenko, and U. Nowak, Phys. Rev. B 86, 214416 (2012). 